\title{
An Atypical Cause of Abdominal Pain in an Elderly Patient
}

\author{
Yaroslav Andrew Jakymec'1, Cara Sherman1, Michelle Annabi-Rabadi², Majed Samarneh², \\ Hanady Zainah ${ }^{2 *}$
}

${ }^{1}$ Lake Erie College of Osteopathic Medicine, Erie, Pennsylvania, USA

${ }^{2}$ Department of Medicine, St. John's Riverside Hospital, Yonkers, New York, USA

Email: ^hanadyzainah@yahoo.com

How to cite this paper: Jakymec, Y.A., Sherman, C., Annabi-Rabadi, M., Samarneh, M. and Zainah, H. (2021) An Atypical Cause of Abdominal Pain in an Elderly Patient. $O$ pen Journal of Nephrology, 11, 370-378. https://doi.org/10.4236/ojneph.2021.113030

Received: June 27, 2021

Accepted: August 16, 2021

Published: August 19, 2021

Copyright $\odot 2021$ by author(s) and Scientific Research Publishing Inc. This work is licensed under the Creative Commons Attribution International License (CC BY 4.0).

http://creativecommons.org/licenses/by/4.0/

\begin{abstract}
Background: Henoch-Schonlein Purpura (HSP), or IgA vasculitis, is the most common vasculitis in children and is characterized by four symptoms: palpable purpura with no concurrent thrombocytopenia or coagulopathy, arthritis/ arthralgias, abdominal pain, and renal disease. HSP does occur in older patients at a much lower frequency. Objective: To discuss the atypical presentation of HSP in an elderly adult and the increased nephrogenic risks that are involved in this patient group. Case Presentation: We present the history, physical findings, laboratory results, and the clinical course of a 74-year-old Caucasian male with an atypical presentation of HSP. Our patient presented with severe gastrointestinal and cutaneous symptoms which occurred after the onset of abdominal pain. Biopsies demonstrated leukocytoclastic vasculitis in the skin and acute inflammation and hemorrhage in the gastrointestinal tract. The diagnosis was confirmed as IgA vasculitis following the renal biopsy. The patient's symptoms improved with the administration of methylprednisolone. Conclusion: In adults with HSP there is greater concern regarding renal involvement and a greater risk of developing end-stage renal disease compared to children. The importance of recognizing HSP in the elderly is imperative for early diagnosis and appropriate treatment to limit renal damage.
\end{abstract}

\section{Keywords}

Henoch-Schonlein Purpura, IgA Vasculitis, Leukocytoclastic Vasculitis

\section{Introduction}

Henoch-Schonlein Purpura (HSP) is an immune-mediated vasculitis associated with IgA deposition. Its etiology is unknown and may be caused by a variety of 
triggers such as infection, medication, malignancy, etc. [1]. IgA vasculitis is the most common vasculitis of childhood, and its prevalence decreases with age. The most commonly involved organ systems include the skin, the gastrointestinal tract, the kidneys, and joints. Characteristic findings of HSP include leukocytoclastic vasculitis along with Immunoglobulin A deposition in the affected organs.

Purpura is the presenting symptom in approximately $75 \%$ of patients, typically preceding other symptoms by around four days [2]. The rash is often localized to gravity/pressure-dependent areas such as the lower extremities. Arthralgias occur in up to $84 \%$ of patients [3] and the arthritis is typically migratory, oligoarticular, and non-deforming. The hips, knees, and ankles are most commonly affected, often with accompanying swelling. A retrospective study of 261 patients diagnosed with HSP conducted from December 1991 to December 2001 found that $58 \%$ of patients reported abdominal pain [4]. Meanwhile, a retrospective review of HSP cases in France found that $48 \%$ of patients presented with gastrointestinal symptoms [5]. However, a case series in India found that only $10 \%$ of adults had abdominal pain upon presentation [6]. In comparison to adults, gastrointestinal symptoms occur in up to $50 \%$ of children with HSP and typically develop within eight days after the appearance of the rash. Renal involvement is more prevalent in older children and adults [7], with the most common finding being hematuria. Kidney involvement typically occurs within a few days up to one month after systemic symptoms arise. The findings on renal biopsy are identical to what is seen in IgA nephropathy.

There are no diagnostic tests for HSP, thus it is important to remain suspicious when this tetrad of organ systems is involved. In this report, we will discuss the case of a 74-year-old male presenting with an atypical sequence of symptoms making it challenging to correctly identify HSP.

\section{Case Presentation}

A 74-year-old Caucasian male with a medical history of coronary artery disease, Hepatitis B, and hypertension presented to the emergency department with 3 days of a severe, 10/10 in intensity, cramping abdominal pain. He denied nausea, vomiting, or diarrhea and endorsed a petechial rash on the dorsum of both feet (Figure 1). The patient stated that the petechial rash appeared about 4 hours after the onset of the abdominal pain. He also endorsed diffuse myalgia. Several weeks prior, the patient had cold-like symptoms that included cough, rhinorrhea, and progressive myalgia. The patient denied any illicit substance, alcohol, or tobacco use. The patient's only home medication was lisinopril. About 6 days prior to admission, the patient was evaluated in the Emergency Department where a CT scan demonstrated concentric wall thickening of the ileum, and a small amount of fluid in the paracolic and rectovesical spaces. He was discharged from the Emergency Department with a 7-day course of metronidazole and ciprofloxacin.

On the physical exam sparse and faint petechiae were noted on the top of the feet as seen in Figure 1. The patient was tender to palpation of the left and right 


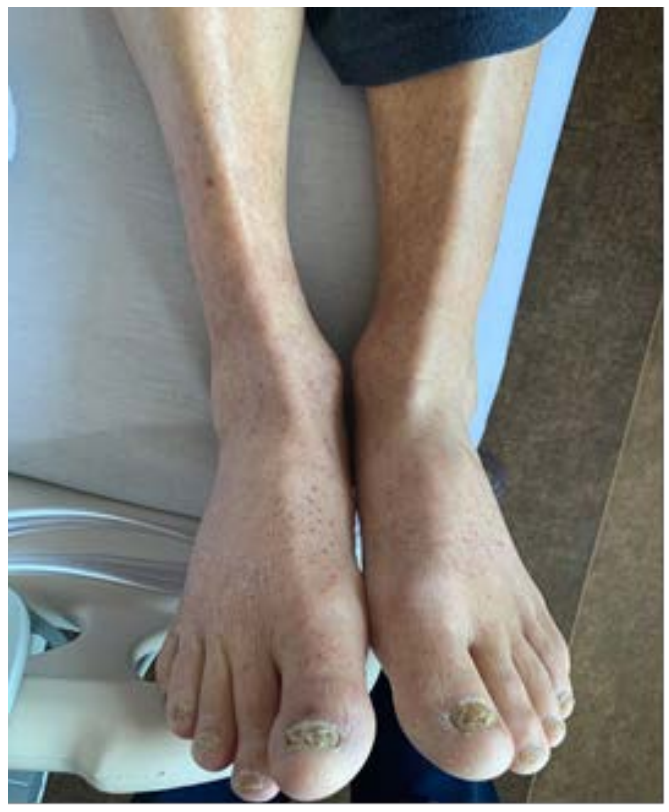

Figure 1. Initial presentation of the palpable purpura.

lower abdominal quadrants. At the time of admission, the patient's $\mathrm{CBC}$ was unremarkable except for a WBC of $10.7 \times 10^{3} / \mathrm{mm}^{3}$ (reference range: 4 to $10 \times$ $10^{3} / \mathrm{mm}^{3}$ ). CMP was only notable for a transaminitis with an AST of 54 (reference range: 15 to $37 \mathrm{U} / \mathrm{L}$ ) and an ALT of 76 (reference range: 13 to $61 \mathrm{U} / \mathrm{L}$ ). The $\mathrm{BUN} / \mathrm{Cr}$ ratio was 10.8/0.7. There was a positive FOBT. During the course of his hospital admission, the patient's UA was notable for microscopic hematuria (Table 1). CRP was elevated at 8.3 (reference range: $0.00-0.03 \mathrm{mg} / \mathrm{dL}$ ) and ESR was within normal ranges. Additional labs including ANA, Proteinase 3, C-ANCA, P-ANCA, atypical P-ANCA, myeloperoxidase Ab, dsDNA, glomerular base membrane $\mathrm{Ab}$, Complement $\mathrm{C} 4$, and Total complement ( $\mathrm{CH} 50)$ were all within normal ranges. Complement $\mathrm{C} 3$ was borderline low at 80 (reference range: 82 - 167 $\mathrm{mg} / \mathrm{dL}$ ). An abdominal CT scan demonstrated distal jejunum concentric wall thickening suggestive of acute enteritis which was new when compared to a previous CT scan. At the time, differentials included enteritis of either inflammatory or infectious cause and as a result, Flagyl, Rocephin, and IV fluids were initiated. Subsequent CTA found no obvious occlusion in the mesenteric vessels but the distal branches were not visualized. A biopsy was taken of the skin lesions noted above.

With the resolution of the previously mentioned symptoms, the patient was discharged home with information for proper outpatient follow up.

Shortly following the noted hospital course, the patient returned to the Emergency Department with a recurrence of severe abdominal pain. The patient continued to have proteinuria and microscopic hematuria on a repeat urinalysis (Table 1). Another CT scan demonstrated acute colitis at the level of the cecum and ascending colon. A colonoscopy demonstrated hemorrhagic mucosa was found in the terminal ileum. Biopsy results from the terminal ileum demonstrated moderate 
Table 1. Urinary analysis results.

\begin{tabular}{cccc}
\hline Date & $2 / 25$ & $03 / 02$ & $3 / 12$ \\
Urine color & Yellow & Yellow & Yellow \\
$\mathrm{pH}$ & 5.5 & 7 & 7 \\
Urine protein & $2+$ & Trace & $2+$ \\
Urine glucose & Negative & Negative & Negative \\
Urine ketone & Negative & Negative & Negative \\
Urine blood & $3+$ & $2+$ & $3+$ \\
Urine nitrate & Negative & Negative & Negative \\
Urine bilirubin & Negative & Negative & Negative \\
Leukocyte esterase & Negative & Negative & Negative \\
Urine WBC & 19 & 6 & 40 \\
Urine RBC & 68 & 275 & 1210 \\
Urine casts & 5 & 2 & 15 \\
U epithelial & 9 & 3 & 21 \\
Urine bacteria & 163 & 25 & 31 \\
\hline
\end{tabular}

acute inflammation with extravasated red blood cells and hemorrhage within the lamina propria, focal erosions, and regenerative/reactive changes. At this time, the skin biopsy performed on the prior admission resulted in leukocy-toclastic vasculitis.

During this admission, the patient initially presented with petechiae similar to his previous admission. As the hospital stay progressed, the purpura coalesced as seen in Figure 2. To further investigate the microscopic hematuria, cystoscopy was performed showing a normal bladder and urethra. To further investigate the hematuria and proteinuria on the UA, a renal biopsy was performed. Renal biopsy results further described below lead to the diagnosis of IgA vasculitis. Figure 3 demonstrates cellular crescents which correspond to glomerular injury within the context of this disease. Upon immunofluorescence microscopy (as seen in Figure 4), IgA deposits within the glomerular capillary walls are noted, as well as in the mesangium. Electron dense deposits are seen on electron microscopy in Figure 5. These findings, particularly those seen in Figure 4, are strongly suggestive of HSP. The patient was treated for the acute exacerbation with methylprednisolone to help shorten the duration of his acute pain symptoms, and discharged with appropriate outpatient follow ups. Unfortunately, despite having several appointments, the patient failed to follow up in the clinic.

\section{Discussion}

Henoch-Schonlein Purpura (HSP) is a systemic, autoimmune, small vessel vasculitis with multiorgan involvement that is associated with IgA deposition. HSP is the 


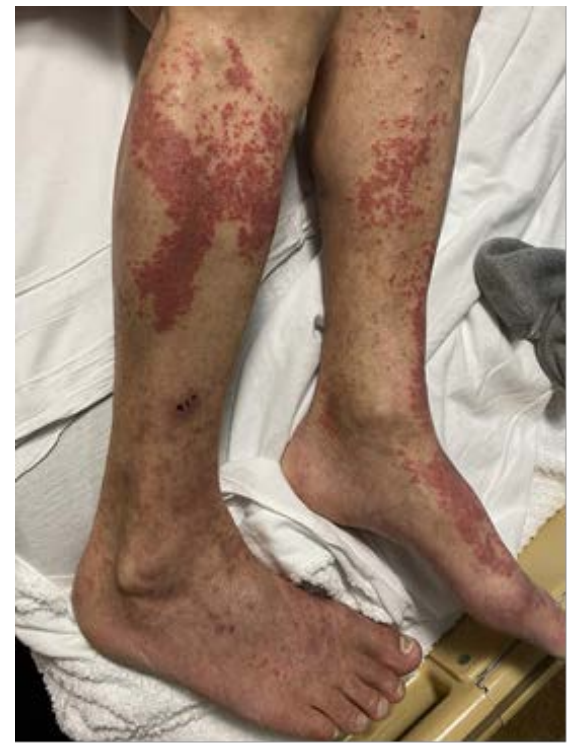

Figure 2. Progression of palpable purpura with coalescent lesions in lower limbs.

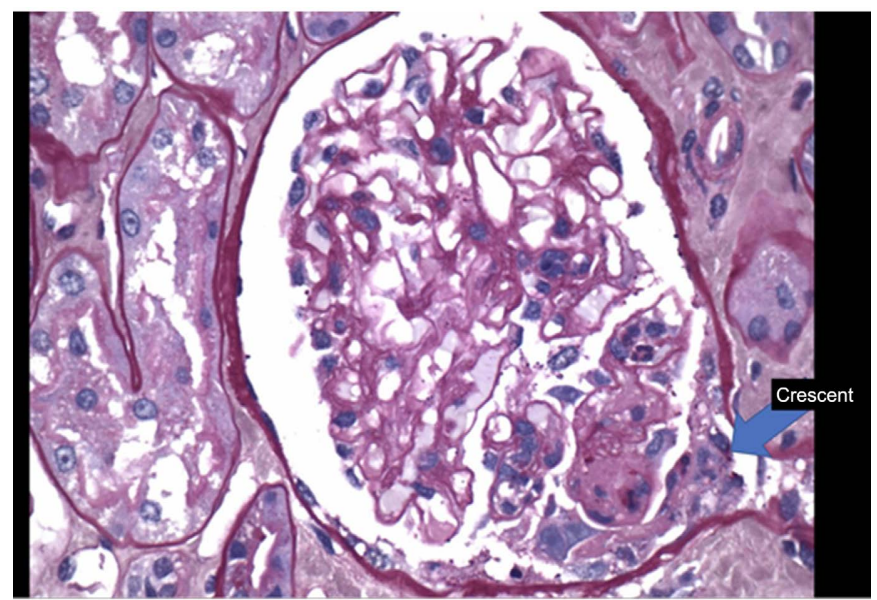

Figure 3. IgA nephropathy with cellular crescent (blue arrow) on light microscopy (Periodic Acid Stain, original magnification $\times 600$ ). Courtesy of Michael B Stokes MD.

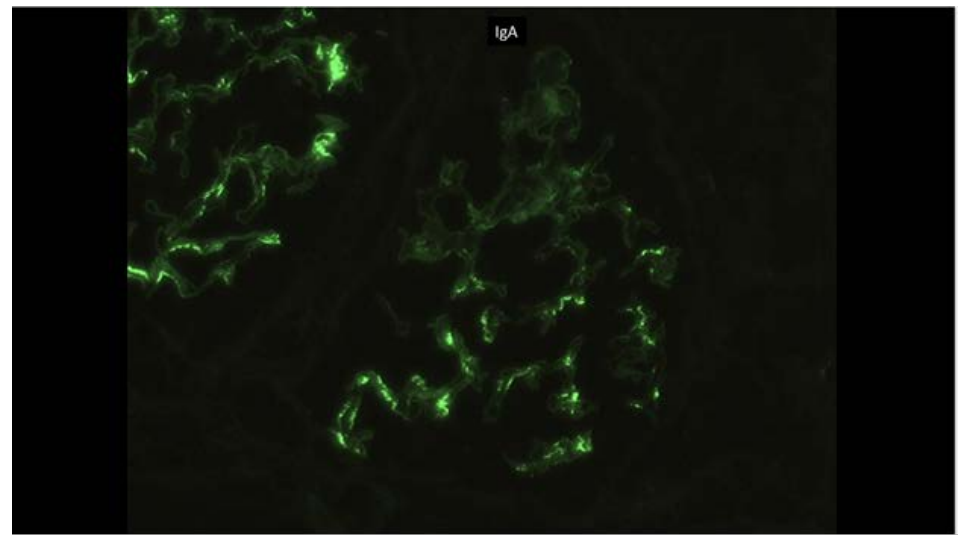

Figure 4. Below showing granular staining for IgA deposits seen in mesangial regions and a few glomerular capillary walls (immunofluorescence, original magnification $\times 400$ ) Courtesy of Michael B Stokes MD. 


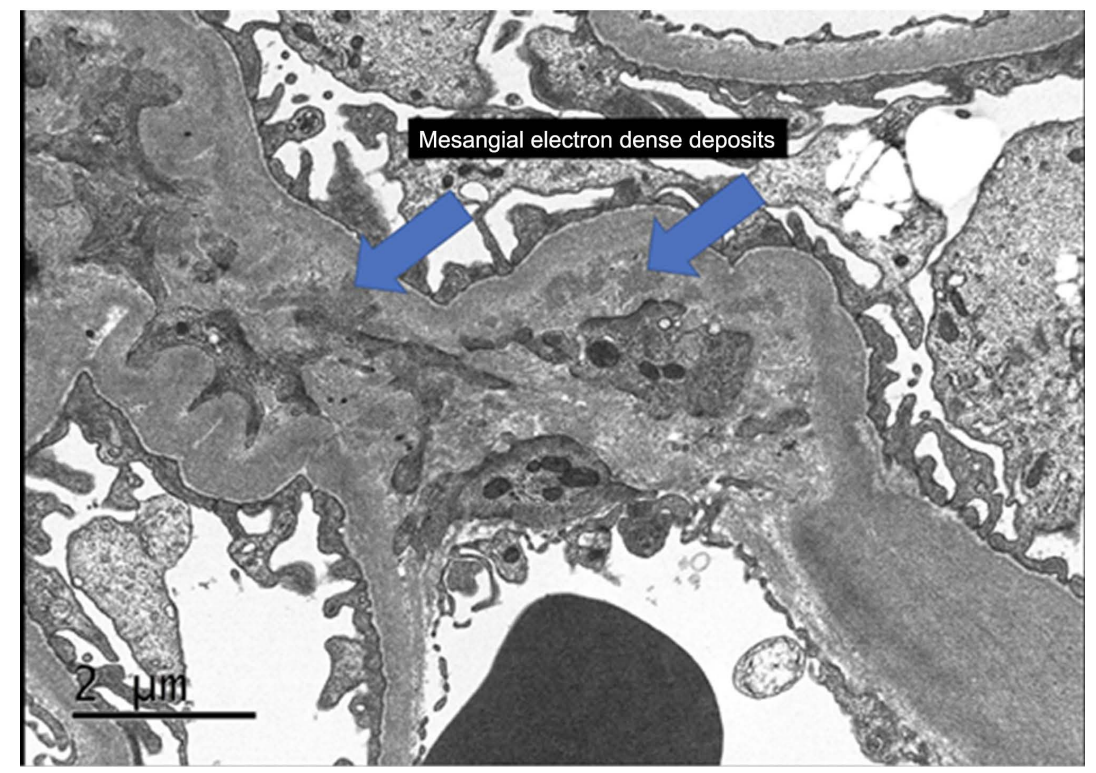

Figure 5. Electron microscopy showing dense deposits (arrows) scattered throughout the mesangium. Courtesy of Michael B Stokes MD.

most common systemic vasculitis in children, as $90 \%$ of cases occur in the pediatric age group [8]. It is typically self-limited, and is characterized by palpable purpura, arthralgia, abdominal pain, and renal involvement. Infections, medications, vaccinations, and malignancies have been implicated as possible triggers for HSP, yet the underlying etiology is unknown. The pathophysiology of HenochSchonlein Purpura is not fully understood. IgA complexes deposit in the wall of small blood vessels leading to neutrophil accumulation which results in inflammation [3]. Complement C3 receptor lymphocytes may bind to immune complexes contributing to the inflammatory response.

There are no specific laboratory tests to assess for markers of HSP. Often, the following tests are done to exclude other diagnoses: Antinuclear Antibody (ANA), Rheumatoid Factor (RF), C3 and C4, Antineutrophil Cytoplasmic Antibodies (CANCA and P-ANCA). The normal results for these markers in our patient excluded some of the more common causes of vasculitis in adults, and led us to continue to investigate more rare causes for our patient's symptoms, including HSP.

The clinical presentation varies, but a palpable purpuric rash is the presenting sign in most cases. The rash often begins as erythematous, macular, or urticarial wheels and is nonpainful. The initial rash can evolve into ecchymoses, petechiae, or palpable purpura. It appears in groups and is symmetrically distributed, typically seen in the lower extremities and buttocks, though the face, trunk, and upper extremities may be involved. There may be associated edema of the hands, feet, or face as well. When the rash is biopsied, leukocytoclastic vasculitis is identified, characterized by neutrophilic infiltration and IgA deposition in the dermis, as demonstrated in our patient.

Arthralgias occur in up to $84 \%$ of patients [3], and are usually migratory, oligoarticular, and nondestructive. Lower extremity joints are more commonly involved 
than joints in the upper extremities. Often, there is prominent swelling and tenderness with considerable limitation of mobility. Our patient, however, did not present with this classic and quite prevalent symptom.

Abdominal pain associated with HSP is caused by submucosal hemorrhage and edema due to vasculitis involving the splanchnic circulation [4]. The inflammation and hemorrhage of the mucosa found on the intestinal biopsies strongly indicate ischemic changes due to small vessel vasculitis in the small branches supplying the intestine. The case for small vessel vasculitis was further supported by the inability to visualize the terminal branches of the mesenteric arteries on the CTA. Gastrointestinal symptoms may range from mild to severe. Mild symptoms consist of nausea, vomiting, and abdominal pain, while severe findings consist of hemorrhage, bowel ischemia, intussusception, or bowel perforation. Intussusception is the most common GI complication of HSP. Gastrointestinal symptoms typically develop about one week after the rash, although that is not always the case, as seen with our patient. On endoscopy, erythema, edema, ulcers, and skip lesions may be seen. The second portion of the duodenum is most commonly involved, although lesions may also be seen in the gastric antrum, cecum, ileum, and colon [9].

IgA containing immune complexes get deposited in glomerular capillaries, leading to renal manifestations. The most common presentation is microscopic hematuria with or without proteinuria [10]. In the case of our patient, during both of his admissions he had significant levels of proteinuria and hematuria. Involvement of the kidneys is more prevalent in adults than in children, and extensive renal involvement is associated with an increased risk of progressive disease. Deterioration of kidney function may ensue over time, resulting in end-stage renal disease. Our patient had a creatinine level between 0.7 and 0.8 during both admissions. On kidney biopsy, granular IgA type 1 and $\mathrm{C} 3$ deposits in the mesangium which supported the diagnosis of IgA Vasculitis. Severity can range from isolated mesangial proliferation to severe crescentic glomerulonephritis. It is also possible for fluorescence to show IgG, IgM, fibrinogen, and C3 in the glomeruli.

Treatment is primarily supportive in most cases. This involves providing adequate hydration, monitoring kidney function/GI involvement, symptomatic treatment of arthralgias, and discontinuing medications suspected of precipitating HSP. Joint pain may be relieved with NSAIDs such as ibuprofen, acetaminophen, naproxen, etc. Studies have shown that glucocorticoid therapy helps shorten the duration of abdominal pain in patients with HSP but does not affect morbidity [11].

In our patient, his clinical presentation arose several weeks after an upper respiratory infection as is seen in approximately one-half of the cases of HSP [12]. This connection, along with the seasonal nature of HSP cases, which primarily occur in the winter, spring and fall but rarely in the summer [13], support a possible association between infections and HSP. The patient's presentation which occurred during the winter is in line with this association. However, compared to children, adults with this disease rarely have abdominal pain as the first clini- 
cal manifestation [6]. In adults, it is more common to have a poor renal outcome than in children [5]. Although our patient had microscopic hematuria and proteinuria, he had normal renal function throughout his hospital stay. The patient was again given several follow up appointments, however the patient failed to return for his follow up appointments.

\section{Conclusion}

Henoch-Schonlein Purpura is not commonly seen in adult populations, but should be suspected when there is gastrointestinal, skin, joint, and/or renal involvement. The typical presenting signs of HSP are usually either a skin rash or arthralgias, and although a vast majority of patients will present with either one of these two symptoms, it is important to be aware of non-classic presentations. Our patient's presentation was atypical in that he presented with severe abdominal pain and a faint petechial rash that led to the diagnosis of HSP and associated renal IgA vasculitis.

\section{Consent}

Written informed consent was obtained from the patient for publication of this case report and any accompanying images. A copy of the written consent is available for review by the Editor-in-Chief of this journal.

\section{Conflicts of Interest}

The authors declare no conflicts of interest regarding the publication of this paper.

\section{References}

[1] Rigante, D., Castellazzi, L., Bosco, A. and Esposito, S. (2013) Is there a Crossroad between Infections, Genetics, and Henoch-Schönlein Purpura? Autoimmun Reviews, 12, 1016-1021. https://doi.org/10.1016/j.autrev.2013.04.003

[2] Jauhola, O., Ronkainen, J., Koskimies, O., et al. (2010) Clinical Course of Extrarenal Symptoms in Henoch-Schonlein Purpura: A 6-Month Prospective Study. Archives of Disease in Childhood, 95, 871-876. https://doi.org/10.1136/adc.2009.167874

[3] Trapani, S., Micheli, A., Grisolia, F., Resti, M., Chiappini, E., Falcini, F. and De Martino, M. (2005) Henoch Schonlein Purpura in Childhood: Epidemiological and Clinical Analysis of 150 Cases over a 5-Year Period and Review of Literature. Seminars in Arthritis and Rheumatism, 35, 143-153. https://doi.org/10.1016/j.semarthrit.2005.08.007

[4] Chang, W.L., Yang, Y.H., Lin, Y.T. and Chiang, B.L. (2004) Gastrointestinal Manifestations in Henoch-Schönlein Purpura: A Review of 261 Patients. Acta Paediatrica, 93, 1427-1431. https://doi.org/10.1111/j.1651-2227.2004.tb02623.x

[5] Pillebout, E., Thervet, E., Hill, G., Alberti, C., Vanhille, P. and Nochy, D. (2002) Henoch-Schönlein Purpura in Adults: Outcome and Prognostic Factors. Journal of the American Society of Nephrology, 13, 1271-1278. https://doi.org/10.1097/01.ASN.0000013883.99976.22

[6] Gupta, V., Aggarwal, A., Gupta, R., Chandra, C.A., Agarwal, V., Lawrence, A. and Mis- 
ra, R. (2018) Differences between Adult and Pediatric Onset Henoch-Schonlein Purpura from North India. International Journal of Rheumatic Diseases, 21, 292-298. https://doi.org/10.1111/1756-185X.13221

[7] Ghrahani, R., Ledika, M.A., Sapartini, G., Setiabudiawan, B. (2014) Age of Onset as a Risk Factor of Renal Involvement in Henoch-Schönlein Purpura. Asia Pacific Allergy, 4, 42-47. https://doi.org/10.5415/apallergy.2014.4.1.42

[8] Watts, R.A., Carruthers, D.M. and Scott, D.G. (1995) Epidemiology of Systemic Vasculitis: Changing Incidence or Definition? Seminars in Arthritis and Rheumatism, 25, 28-34. https://doi.org/10.1016/S0049-0172(95)80015-8

[9] Oshikata, C., Tsurikisawa, N., Takigawa, M., et al. (2013) An Adult Patient with Henoch-Schönlein Purpura and Non-Occlusive Mesenteric Ischemia. BMC Research Notes, 6, Article No. 26. https://doi.org/10.1186/1756-0500-6-26

[10] Chang, W.L., Yang, Y.H., Wang, L.C., Lin, Y.-T. and Chiang, B.-L. (2005) Renal Manifestations in Henoch-Schönlein Purpura: A 10-Year Clinical Study. Pediatric Nephrology, 20, 1269-1272. https://doi.org/10.1007/s00467-005-1903-Z

[11] Rosenblum, N.D. and Winter, H.S. (1987) Steroid Effects on the Course of Abdominal Pain in Children with Henoch-Schönlein Purpura. Pediatrics, 79, 1018-1021.

[12] Levy, M., Broyer, M., Arsan, A., et al. (1976) Anaphylactoid Purpura Nephritis in Childhood: Natural History and Immunopathology. Advances in Nephrology from the Necker Hospital, 6, 183-228.

[13] Hwang, H.H., Lim, I.S., Choi, B.S. and Yi, D.Y. (2018) Analysis of Seasonal Tendencies in Pediatric Henoch-Schönlein Purpura and Comparison with Outbreak of Infectious Diseases. Medicine (Baltimore), 97, e12217. https://doi.org/10.1097/MD.0000000000012217 